\title{
Histomorphological Assessment of Formalin versus Nonformalin Fixatives in Diagnostic Surgical Pathology
}

\author{
Jayaprakash Kubalady Shetty ${ }^{1}$ Hannah Fathima Babu ${ }^{1}$ Kishan Prasad Hosapatna Laxminarayana ${ }^{1}$ \\ ${ }^{1}$ Department of Pathology, K. S. Hegde Medical Academy, \\ Nitte (Deemed to be University), Mangaluru, Karnataka, India

\begin{abstract}
Address for correspondence Kishan Prasad HL, Additional Professor, Department of Pathology, K. S. Hegde Medical Academy, Nitte (Deemed to be University), Mangaluru, Karnataka 575018, India (e-mail: kishanprasadhl@nitte.edu.in).
\end{abstract}

\begin{abstract}
Introduction Fixation is the critical step in the preservation of tissues in diagnostic pathology. The formalin is an economical and excellent fixative with the inherent property of adequate fixation. The well-established side effects of formalin include mucosal irritation, upper respiratory diseases, and corrosive injury to the gastrointestinal tract. In addition, substantial evidence exists regarding the potential role of formaldehyde as a human carcinogen. The carcinogenic and toxic effects of formalin encourage searching for alternative fixatives for tissue fixation. However, "the formalin dogma" has severely hampered the search for alternative fixatives for many years.

Material and Methods Ninety tissues of liver and skeletal muscle obtained during autopsies were immersed in adequate amounts of the following fixatives: formalin (10\%), methyl alcohol (70\%), and acetone (100\%). The comparison among the three was made based on time for fixation, preservation of tissue architecture, cell borders, cytoplasm, nuclear contours, chromatin texture, and uniformity of staining.

Results The tissue preserved in formalin undergoes rapid fixation compared with alcohol and acetone. The tissue architecture, cell border characteristics of alcohol and acetone was found satisfactory compared with formalin. The cytoplasm and nuclear contour were superior with the formalin. The chromatin texture and uniformity of

Keywords

- formalin

- alcohol

- fixatives

- formalin toxicity

- tissue specimen staining were similar with all the three fixatives.

Conclusion The formalin is considered superior to most of the parameters, whereas both methyl alcohol and acetone showed nearly equivalent scores. Hence, owing to the potential human health hazards and carcinogenicity of formalin, no rational reasons hamper the complete substitution of formalin with alternative fixatives such as alcohol and acetone in diagnostic pathology and medical research.
\end{abstract}

\section{Introduction}

In diagnostic pathology, fixation is the critical step in the preparation of histological tissues by which biological tissues are preserved. Neutral-buffered formalin (NBF) (10\%) is used for tissue fixation in the majority of laboratories for

DOI https://doi.org/ $10.1055 / \mathrm{s}-0040-1722546$ ISSN 0974-2727. many years. There is a consensus among the pathologists and researchers that formalin is the cheap and best fixative; hence, there is no need for an alternative to formalin, generating the "the formalin dogma." This approach has severely hampered the search for alternative fixatives. ${ }^{1}$ The well-established side effects of formalin include irritation of eye, nose, throat,

(c) 2020. The Indian Association of Laboratory Physicians.

This is an open access article published by Thieme under the terms of the Creative Commons Attribution-NonDerivative-NonCommercial-License, permitting copying and reproduction so long as the original work is given appropriate credit. Contents may not be used for commercial purposes, or adapted, remixed, transformed or built upon. (https://creativecommons.org/licenses/by-nc-nd/4.0/).

Thieme Medical and Scientific Publishers Pvt. Ltd. A-12, 2nd Floor, Sector 2, Noida-201301 UP, India 
and skin, upper respiratory disease, cough, chest pain, and wheezing. It also causes corrosive injury to the gastrointestinal tract. It can also produce systemic complications like metabolic acidosis, circulatory shock, and acute renal failure..$^{2-4}$ The chromosomal alterations were detected in laboratory health workers who are exposed to formalin., ${ }^{4,5}$ The International Agency for Research on Cancer (IARC) recently reclassified formaldehyde as a human carcinogen that causes nasopharyngeal cancer and leukemia. ${ }^{5}$ Hence, the fixative, which is an alternative to formalin and offers more protection for health workers, is needed in the present scenario.

Methyl alcohol is one of the fixatives, which denatures proteins by replacing water in the environment disrupting hydrophobic and hydrogen bonding. Thus, it alters the tertiary structure and solubility of proteins in water. It is commonly used as a fixative for peripheral blood films. Acetone has a similar action as that of alcohol, and has been used as a fixative and dehydrating agent for tissue processing, particularly rapid hand processing of small specimens. ${ }^{6,7}$

The carcinogenic and toxic potential of formalin is a potential drive to reconsider the formalin dogma and to evaluate the use of alternative fixatives such as alcohol and acetone, which may offer better technical performance and greater protection for health workers. ${ }^{1-6}$ This study was undertaken to assess the efficacy of formalin versus nonformalin fixatives like alcohol and acetone in routine histopathology.

\section{Materials and Methods}

This is a cross-sectional study conducted at the department of pathology of a tertiary hospital of South India. We have compared the two nonformalin fixatives methyl alcohol and acetone to formalin, which is the standard fixative used in tissue fixation in the laboratories for diagnostic histopathology across the world. After obtaining ethical clearance and permission from the Medical Superintendent of the hospital, 90 tissues of skeletal muscle (45) and liver (45) were collected during the autopsy procedure. These tissues are particularly taken, as they are easy to obtain and not easily autolyzed. The fresh tissues are sliced into $2 \times 1 \mathrm{~cm}$ for optimal fixation and are immersed in sufficient amount of 10\% formalin, $70 \%$ methyl alcohol, and 100\% acetone. After adequate fixation, these tissues are subjected to routine histological processing and paraffin tissue blocks were prepared. The time taken for fixation of each tissue immersed in different fixatives is noted. If the tissue is not fixed, it will be friable and hemorrhagic, whereas fixed tissue will be firm. The unfixed tissue is kept for adequate fixation, later they are processed, and paraffin blocks are prepared as per the standard histological processing. The fixed tissues are then analyzed and compared on gross morphology and histopathological characteristics based on hematoxylin and eosin (H\&E) staining (-Fig. 1).

In the H\&E slides, the comparison was made based on the subjective evaluation of seven morphological features: time for fixation, tissue architecture, cell borders, cytoplasm, nuclear contours, chromatin texture, and uniformity

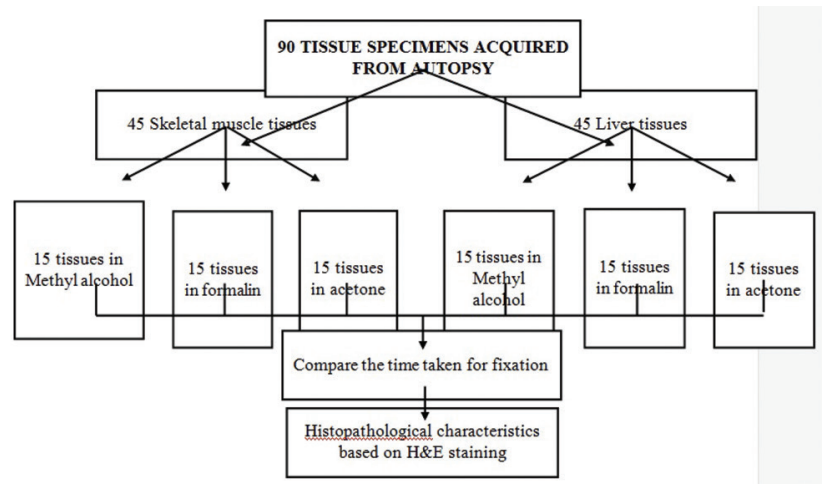

Fig. 1 Flowchart showing methodology followed in the study.

of staining by an expert pathologist. The results of the quality of fixation of each case were graded ( 1 - below average; 2 - average; and 3 - above average). Results were compared. The data about the quality of fixation of the tissues were summarized by using percentages. Fischer's exact test or chi-square test or one-way analysis of variance was used to calculate the $p$-value. A $p$-value of $<0.05$ was considered as statistically significant.

\section{Results}

This was a cross-sectional study conducted at the department of pathology of a tertiary hospital of South India. The procedures followed were in accord with the ethical guidelines established by the institution. Forty-five specimens each of liver and skeletal muscle tissue were procured during autopsies and were fixed using formalin (30), methyl alcohol (30), and acetone (30). The comparison between anatomical tissues fixed with formalin, methyl alcohol, and acetone showed statistically significant variation concerning tissue architecture, cell borders, cytoplasm, and nuclear contour of the tissue ( $p$-value $<0.05$ ). Histopathological images of formalin, alcohol, and acetone fixed liver and skeletal muscle are shown in - Figs. 2-4.

The comparison of time taken for fixation showed that there is a significant difference $(p=0.000)$ in average time for fixation for formalin (24 hours), methyl alcohol (60 hours), and acetone (97.2 hours) (-Table $\mathbf{1}$ ).

Comparison of tissue architecture among three fixatives showed that there was a significant difference in grading $(p=0.001)$. The tissue architecture was graded above average in all the tissues when formalin and methyl alcohol was used as fixatives. Whereas when the tissues were fixed with acetone tissue architecture was graded above average for $60 \%$ and average for $40 \%$ (- Table 2 ).

There was a significant difference in cell borders of tissues fixed by the three fixatives $(p=0.009)$. The cell borders were above average in all the tissues of formalin and methyl alcohol fixation but were above average in $80 \%$ with acetone.

The comparison of cytoplasmic features among tissues showed that there was a significant difference in grading among the three fixatives $(p=0.001)$. The cytoplasm features were above average in all the tissues with formalin. In the 

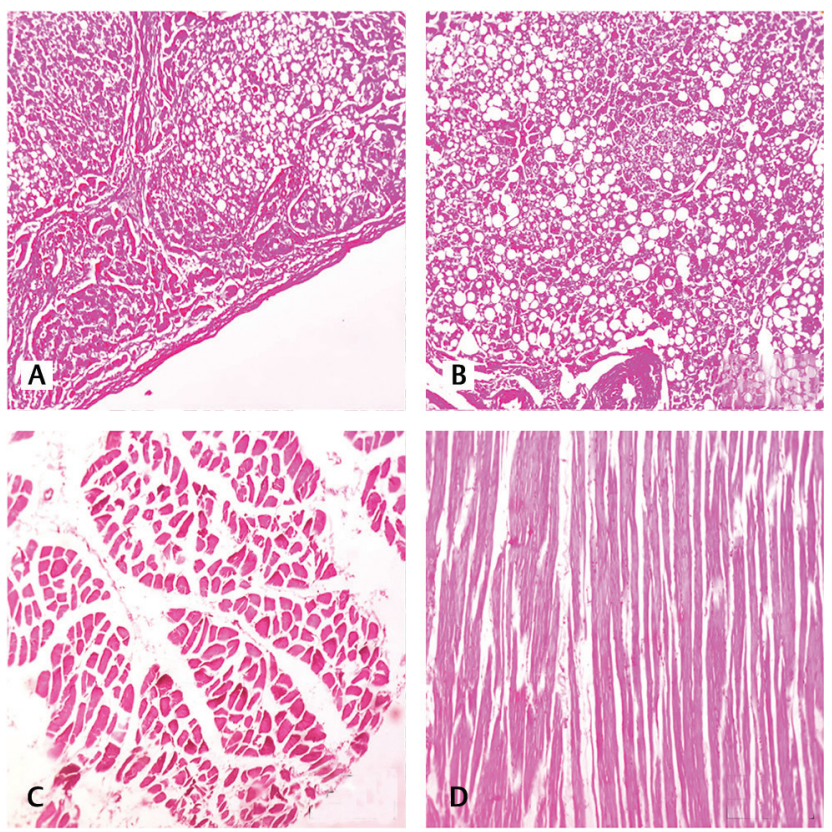

Fig. 2 (A-D) Histopathology showing formalin fixed liver and muscle tissue (hematoxylin and eosin $[H \& E], \times 100)$.
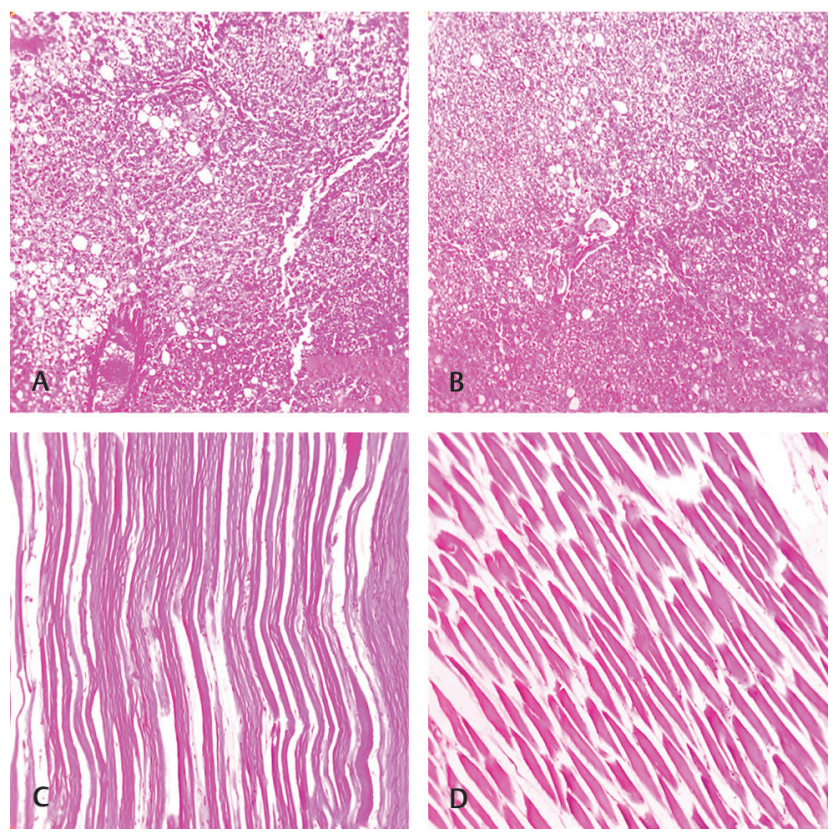

Fig. 3 (A-D) Histopathology showing alcohol fixed liver and muscle tissue (hematoxylin and eosin $[\mathrm{H} \& \mathrm{E}], \times 100)$. methyl alcohol and acetone fixed tissues, cytoplasmic features were graded above average and average, respectively (50\% each). There was a significant difference in nuclear contour when fixed with different fixatives $(p=0.001)$. The nuclear contour with formalin was above average in $40 \%$ tissue and in the remaining $60 \%$ tissue it was graded average as well as below average ( $30 \%$ each). When tissues were fixed with methyl alcohol, the nuclear contour was average in $60 \%$ and below average in $40 \%$ tissues. Nuclear contour was graded above average and average, $35 \%$ each, when fixed with acetone, whereas the remaining 30\% tissue was below average (-Table 2).
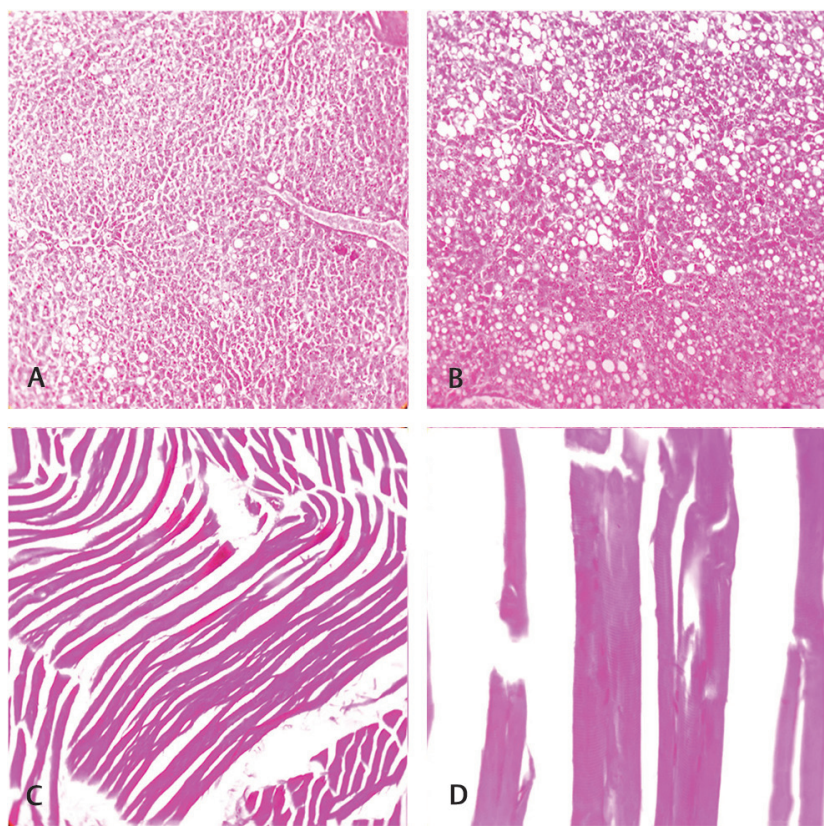

Fig. 4 (A-D) Histopathology showing acetone fixed liver and muscle tissue (hematoxylin and eosin [H\&E], × 100).

Table 1 Time for fixation

\begin{tabular}{|l|l|l|}
\hline Fixative & $\begin{array}{l}\text { Mean time of } \\
\text { fixation (h) }\end{array}$ & $\begin{array}{l}\text { p-Value } \\
\text { (one-way ANOVA } \\
\text { test) }\end{array}$ \\
\hline Formalin & 24 & \\
\hline Methyl alcohol & 60 & 0.000 \\
\hline Acetone & 97.2 & \\
\hline
\end{tabular}

Abbreviation: ANOVA, analysis of variance.

Table 2 Tissue architecture and nuclear contour

\begin{tabular}{|l|l|l|l|l|l|}
\hline & Grading & Formalin (\%) & Methyl alcohol (\%) & Acetone (\%) & p-Value \\
\hline \multirow{4}{*}{ Tissue architecture } & Above average & 100 & 100 & 60 & $<0.001$ \\
\cline { 2 - 5 } & Average & 0 & 0 & 40 & \multirow{2}{*}{$<0.001$} \\
\cline { 2 - 5 } & Below average & 0 & 0 & 0 & 35 \\
\hline \multirow{3}{*}{ Nuclear contour } & Above average & 40 & 0 & 35 & 30 \\
\cline { 2 - 5 } & Average & 30 & 60 & 40 & 30 \\
\cline { 2 - 5 } & Below average & 30 & 40 & \\
\hline
\end{tabular}


The chromatin texture of tissue fixed with formalin and methyl alcohol was graded above average and average, respectively ( $50 \%$ each). Whereas when tissues were fixed with acetone, $65 \%$ was above average and $35 \%$ average. The uniformity of staining was above average and average with formalin and methyl alcohol, respectively (50\% each). With acetone, uniformity of staining was above average for $70 \%$ of tissues and average for 30\% tissues. The chromatin texture and uniformity of staining of the tissues fixed by formalin, methyl alcohol, and acetone were not statistically significant.

\section{Discussion}

An optimal fixative should be nontoxic, cost-effective, and enable a detailed morphological analysis with high-quality histochemical and immunohistochemical staining with preservation of deoxyribonucleic acid (DNA) and ribonucleic acid (RNA). Since the fixative with such features does not exist, it is essential to explore the existing as well as new fixatives. ${ }^{5,7}$

In diagnostic pathology, NBF was considered as the "gold standard" over the years. It is cheap, enables long-term storage, preserves morphological features, and allows reliable histochemical analysis. However, formaldehyde was classified as a carcinogen by the IARC, and therefore there is an impending risk to individuals who handles the formalin solution. ${ }^{5,7}$ Many studies have reported that the less toxic alcohol-based cross-linking fixatives (F-Solv) and noncross-linking fixatives (Boonfix and RCL2) are comparable to NBF. They were found to be suitable for fixation of tissue although better results were observed with NBF. The lower performance of Boonfix and RCL2 was attributed to pepsin AR, which caused significant tissue damage. The omission of pepsin AR resulted in better immunostaining. ${ }^{8}$ The differences compared with formalin fixation was evident in alcohol-based fixatives, mainly restricted to higher stain affinity and considerable tissue shrinkage. The alcohol-based fixatives are known to have higher stain affinity and caused considerable tissue shrinkage when compared with formalin fixation. However, nuclear detail and RNA extraction are better visualized with alcohol-based fixatives..$^{9,10}$ The reported advantages of noncross-linking alcohol-based fixatives include faster fixation, elimination of carcinogenic vapors, better preservation of glycogen, DNA, and RNA. In contrast, the variability of tissue staining, tissue shrinkage and hardening, partial or complete lysis of erythrocytes, and increased flammability is the disadvantages that hinder alcohol fixative usage. ${ }^{10,11}$ Another alternative fixatives used along with the alcohol is acetic acid (such as in RCL2). Acetic acid complements the action of ingredients such as alcohol, makes collagen fibers swell, precipitates nucleoprotein, and has a solvent action on cytoplasmic granules. ${ }^{11,12}$

Compared with previous studies, the present study showed that formalin is a superior fixative under all parameters followed by alcohol and then acetone. However, both acetone and alcohol showed acceptable preservation of tissue morphology. The tissue architecture and cell borders in both formalin- and alcohol-preserved tissue was well maintained compared with acetone. Formalin was the preferred choice to alcohol and acetone about cytoplasmic tissue characters and nuclear contour. This difference is due to the mechanism of action of each fixative. There are two broad categories of fixatives: coagulant fixatives and noncoagulant fixatives (cross-linking). Alcohol and acetone (coagulative fixatives) are thought to form a porous meshwork of protein strands. They act as dehydrants and denatures as well as precipitates protein. Although a significant component of cell membranes, cytoskeletons are formed by lipoproteins and fibrous proteins, and coagulation of proteins protect the tissue architecture from degrading. ${ }^{6-8}$ The formalin (cross-linking fixative) joins proteins with other proteins as well as nucleic acids by cross-linking and cross-links nucleic acids with each other. This stabilizes the tissue architecture for histological evaluation..$^{12}$ The chromatin texture and uniformity of staining with all three fixatives appeared similar. The mean time of fixation was highest for acetone, followed by alcohol and then formalin. The anatomical tissue preserved in formalin undergoes rapid fixation compared with alcohol and acetone. These newer fixatives are less toxic than formalin, but the majority of them are inflammable, and they do contain components that are potentially toxic for humans. ${ }^{9-11}$ The fixation, the embedding procedure, the infrastructure and logistics needed for fixation, storage, and the associated costs can be different depending on the composition of the fixatives. Formalin is cost-effective, readily available when compared with other fixatives like alcohol and acetone. Hence, it is widely used. Therefore, as an alternative or to second formalin fixation methyl alcohol gives nearly equivalent scores to formalin and can be used as an alternative fixative followed by acetone. ${ }^{8-12}$ Further studies are required with a larger sample size with added criteria to further authenticate the observation and conclusion of the present research for clinical use. In addition, there is a necessity for similar studies to look for the effect of these fixatives on immunohistochemistry.

\section{Funding}

This study was funded by ICMR for Short Term Studentship project.

\section{Conflict of Interest}

None declared.

\section{Acknowledgments}

This study was done as a part of the ICMR project. We would like to thank ICMR for the funding.

\section{References}

1 Bardana EJ Jr, Montanaro A. Formaldehyde: an analysis of its respiratory, cutaneous, and immunologic effects. Ann Allergy 1991;66(6):441-452

2 Bostwick DG, al Annouf N, Choi C. Establishment of the formalin-free surgical pathology laboratory. Utility of an alcohol-based fixative. Arch Pathol Lab Med 1994;118(3): 298-302

3 Carleton HM, Drury RAB, Wallington EA, Carleton's Histological Technique. 5th ed. Oxford; New York: Oxford University Press; 1980:520 p 
4 Cogliano VJ, Grosse Y, Baan RA, Straif K, Secretan MB, ElGhissassiF; WorkingGroupforVolume88.Meetingreport:summary of IARC monographs on formaldehyde, 2-butoxyethanol, and 1-tert-butoxy-2-propanol. Environ Health Perspect 2005;113(9):1205-1208

5 Eltoum I, Fredenburgh J, Myers RB, Grizzle WE. Introduction to the theory and practice of fixation of tissues. J Histotechnol 2001;24(3):173-190

6 Lillie RD, Fullmer HM, Histopathologic Technic and Practical Histochemistry. 4th ed. New York: McGraw-Hill; 1976:942 p

7 Loomis TA. Formaldehyde toxicity. Arch Pathol Lab Med 1979; 103(7):321-324

8 Santovito A, Schilirò T, Castellano S, et al. Combined analysis of chromosomal aberrations and glutathione S-transferase M1 and T1 polymorphisms in pathologists occupationally exposed to formaldehyde. Arch Toxicol 2011;85(10):1295-1302
9 Tbakhi A, Totos G, Hauser-Kronberger C, et al. Fixation conditions for DNA and RNA in situ hybridization: a reassessment of molecular morphology dogma. Am J Pathol 1998;152(1):35-41

10 Thavarajah R, Mudimbaimannar VK, Elizabeth J, Rao UK, Ranganathan K. Chemical and physical basics of routine formaldehyde fixation. J Oral Maxillofac Pathol 2012;16(3):400-405

11 van Essen HF, Verdaasdonk MA, Elshof SM, de Weger RA, van Diest PJ. Alcohol based tissue fixation as an alternative for formaldehyde: influence on immunohistochemistry. J Clin Pathol 2010;63(12):1090-1094

12 Zanini C, Gerbaudo E, Ercole E, Vendramin A, Forni M. Evaluation of two commercial and three home-made fixatives for the substitution of formalin: a formaldehyde-free laboratory is possible. Environ Health 2012;11(1):59 [Internet] 